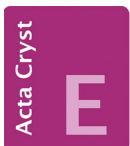

CRYSTALLOGRAPHIC COMMUNICATIONS

ISSN 2056-9890

\section{Supramolecular interactions in 2,6-diamino-4- chloropyrimidin-1-ium 5-chlorosalicylate and bis(2,6-diamino-4-chloropyrimidin-1-ium) naphthalene-1,5-disulfonate}

\author{
Robert Swinton Darious, ${ }^{a}$ Packianathan Thomas Muthiah ${ }^{\mathrm{a} *}$ and Franc Perdih ${ }^{\mathrm{b}}$
}

Edited by M. Zeller, Purdue University, USA

Keywords: crystal structure; hydrogen bonding; supramolecular architecture; halogen-halogen interaction; quadruple array; homosynthon; heterosynthon.

CCDC references: $1817972 ; 1817971$

Supporting information: this article has supporting information at journals.iucr.org/e

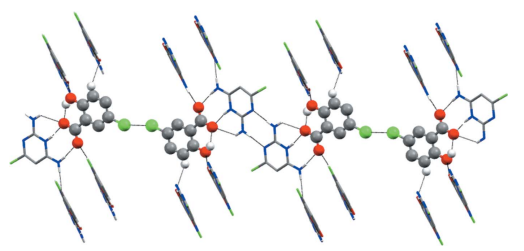

OPEN $\odot$ ACCESS
${ }^{\mathbf{a}}$ School of Chemistry, Bharathidasan University, Tiruchirappalli 620 024, Tamilnadu, India, and ${ }^{\mathbf{b}}$ Faculty of Chemistry and Chemical Technology, University of Ljubljana, Večna, pot 113, PO Box 537, SI-1000 Ljubljana, Slovenia.

*Correspondence e-mail: tommtrichy@yahoo.co.in

The crystals of two new salts, 2,6-diamino-4-chloropyrimidin-1-ium 5-chlorosalicylate, $\mathrm{C}_{4} \mathrm{H}_{6} \mathrm{ClN}_{4}{ }^{+} \cdot \mathrm{C}_{7} \mathrm{H}_{4} \mathrm{ClO}_{3}{ }^{-}$, (I), and bis(2,6-diamino-4-chloropyrimidin-1ium) naphthalene-1,5-di-sulfonate, $2 \mathrm{C}_{4} \mathrm{H}_{6} \mathrm{ClN}_{4}{ }^{+} \cdot \mathrm{C}_{10} \mathrm{H}_{6} \mathrm{O}_{6} \mathrm{~S}_{2}{ }^{2-}$, (II), have been synthesized and characterized by single-crystal $\mathrm{X}$-ray diffraction. In both compounds, the $\mathrm{N}$ atom of the pyrimidine group in between the amino substituents is protonated and the pyrimidinium cation forms a pair of $\mathrm{N}-$ $\mathrm{H}$. . O hydrogen bonds with the carboxylate/sulfonate ion, leading to a robust $R_{2}^{2}(8)$ motif (supramolecular heterosynthon). In compound (I), a selfcomplementary base pairing involving the other pyrimidinium ring nitrogen atom and one of the amino groups via a pair of $\mathrm{N}-\mathrm{H} \cdots \mathrm{N}$ hydrogen bonds $\left[R_{2}^{2}(8)\right.$ homosynthon] is also present. In compound (II), the crystallographic inversion centre coincides with the inversion centre of the naphthalene-1,5disulfonate ion and all the sulfonate $\mathrm{O}$ atoms are hydrogen-bond acceptors, generating fused-ring motifs and a quadruple $D D A A$ array. A halogen-bond $(\mathrm{Cl} \cdots \mathrm{Cl})$ interaction is present in (I) with a distance and angle of 3.3505 (12) $\AA$ and $151.37(10)^{\circ}$, respectively. In addition, a $\mathrm{C}-\mathrm{Cl} \cdots \pi$ interaction and a $\pi-\pi$ interaction in (I) and a $\pi-\pi$ interaction in (II) further stabilize these crystal structures.

\section{Chemical context}

The study of supramolecular interactions in the crystals of pyrimidinium salts continues to be an active field since the pyrimidine fragment is a component of nucleobases and many drug molecules. The pyrimidine group offers two protonation sites (the two ring nitrogens) and the site of protonation depends on the nature of the substituents. Tautomerism of the pyrimidinium cation has also been reported recently (Rajam et al., 2017). The pyrimidinium-carboxylate interaction is also of fundamental importance in biology since it is involved in protein-nucleic acid interactions and drug-receptor recognition (Hunt et al., 1980; Baker \& Santi, 1965). The molecules are often self-assembled by hydrogen bonding, halogen bonding, cation $\cdots \pi$, anion $\cdots \pi$ and $\pi-\pi$ stacking interactions. Among these interactions, halogen bonding is of particular current interest (Cavallo et al., 2016). Various substituted pyrimidines and their interactions with different acids have been studied systematically in our laboratory. The variation in supramolecular architectures resulting from the different substituents in the base and the acid is being investigated, and 
crystal structures of 2,6-diamino-4-chloropyrimidinium salts with carboxylate/sulfonate have been reported recently from our laboratory (Mohana et al., 2017). The same pyrimidine derivative has been used to prepare the title compounds in order to further study the supramolecular architectures and the role of the halogen bond.<smiles></smiles><smiles>O=C(O)c1c(O)cccc1Cl</smiles>

(I)
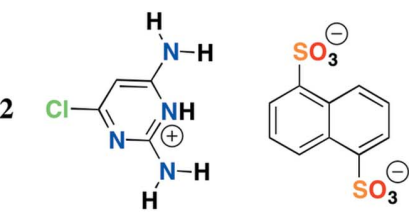

(II)

\section{Structural commentary}

The salt of compound (I) crystallizes with one CDAPY (2,6diamino-4-chloropyrimidinium) cation and one CSA (5chlorosalicylate) anion in the asymmetric unit (Fig. 1). The pyrimidinium cation is protonated at the $\mathrm{N} 1$ position (see Fig. 1 for atom numbering) and this is confirmed by an increase in the internal bond angle. The $\mathrm{C} 2-\mathrm{N} 3-\mathrm{C} 4$ angle at the unprotonated $\mathrm{N} 3$ atom is $115.1(2)^{\circ}$, while for the protonated $\mathrm{N} 1$ atom, the $\mathrm{C} 2-\mathrm{N} 1-\mathrm{C} 6$ angle is $121.8(2)^{\circ}$. The ion-pair (CDAPY and CSA) is almost planar [dihedral angle = $\left.4.22(11)^{\circ}\right]$. The carboxylate group of CSA is twisted slightly with respect to the remainder of the anion [dihedral angle= $\left.3.9(3)^{\circ}\right]$. The salt of compound (II) crystallizes with one CDAPY (2,6-diamino-4-chloropyrimidinium) cation and half a molecule of NSA (naphthalene-1,5-disulfonate) anion in the asymmetric unit (Fig. 2), the other half of NSA being generated by an inversion centre. A crystallographic inversion centre coinciding with the inversion centre of the NSA ion has also been reported earlier (Liu, 2012; Xu, 2012; Liu \& Chen, 2012). The pyrimidinium cation is again protonated at the $\mathrm{N} 1$ position (see Fig. 2 for atom numbering) and this is confirmed by an increase in the internal bond angle. The $\mathrm{C} 2-\mathrm{N} 3-\mathrm{C} 4$ angle at the unprotonated $\mathrm{N} 3$ atom is $115.40(16)^{\circ}$, while the angle at the protonated $\mathrm{N} 1$ atom $(\mathrm{C} 2-\mathrm{N} 1-\mathrm{C} 6)$ is $121.84(16)^{\circ}$. All of the sulfonate oxygen atoms of the NSA

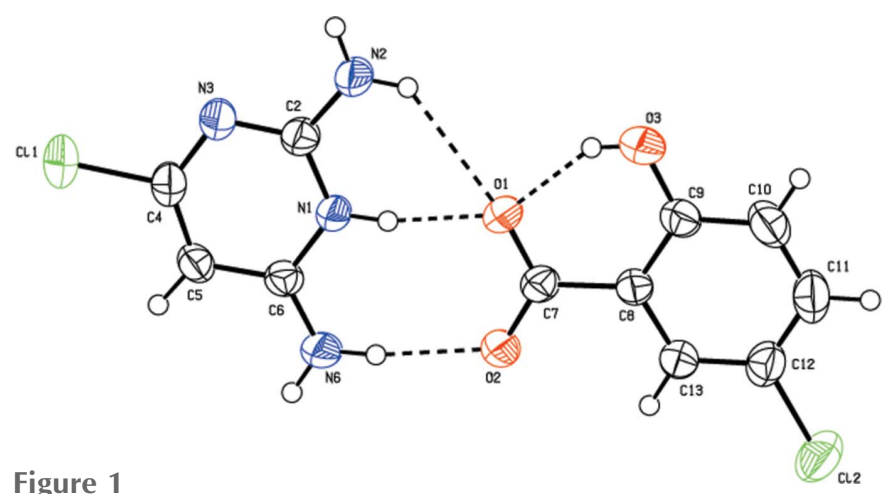

ORTEP view of compound (I) with the atom-numbering scheme. Displacement ellipsoids are drawn at $50 \%$ probability level. Dashed lines represent hydrogen bonds.
Table 1

Hydrogen-bond geometry $\left(\AA,^{\circ}\right)$ for (I).

\begin{tabular}{|c|c|c|c|c|}
\hline$D-\mathrm{H} \cdots A$ & $D-\mathrm{H}$ & $\mathrm{H} \cdots A$ & $D \cdots A$ & $D-\mathrm{H} \cdots A$ \\
\hline $\mathrm{N} 1-\mathrm{H} 1 \cdots \mathrm{O} 1$ & 0.86 & 1.82 & $2.664(3)$ & 168 \\
\hline $\mathrm{N} 2-\mathrm{H} 2 A \cdots \mathrm{O} 1$ & 0.86 & 2.56 & $3.223(3)$ & 135 \\
\hline $\mathrm{N} 2-\mathrm{H} 2 B \cdots \mathrm{N} 3^{\mathrm{i}}$ & 0.86 & 2.13 & $2.970(3)$ & 165 \\
\hline $\mathrm{O} 3-\mathrm{H} 3 \cdots \mathrm{O} 1$ & 0.82 & 1.83 & $2.557(3)$ & 146 \\
\hline $\mathrm{N} 6-\mathrm{H} 6 A \cdots \mathrm{O} 2$ & 0.86 & 1.97 & $2.824(3)$ & 172 \\
\hline $\mathrm{N} 6-\mathrm{H} 6 B \cdots \mathrm{O} 2^{\mathrm{ii}}$ & 0.86 & 1.96 & $2.819(3)$ & 172 \\
\hline $\mathrm{C} 10-\mathrm{H} 10 \cdots \mathrm{O}^{\mathrm{iii}}$ & 0.93 & 2.51 & $3.358(4)$ & 151 \\
\hline
\end{tabular}

anion are involved in hydrogen bonding. The $\mathrm{S} 1-\mathrm{O} 1, \mathrm{~S} 1-\mathrm{O} 2$ and $\mathrm{S} 1-\mathrm{O} 3$ distances are similar [1.4550 (15), $1.4584(15)$ and 1.4431 (16) Å respectively].

\section{Supramolecular features}

In salt (I), the protonated $\mathrm{N} 1$ atom and the amino hydrogen (N6) atom of CDAPY are hydrogen bonded via two N$\mathrm{H} \cdots \mathrm{O}$ bonds (Table 1 ) forming a robust $R_{2}^{2}(8)$ ring motif (heterosynthon) involving the carboxylate group. The typical intramolecular hydrogen-bond $S(6)$ motif (involving the carboxyl group and the phenolic-OH) observed in salicylates/ salicylic acid is also present (Bernstein et al., 1995; Prabakaran et al., 2001; Panneerselvam et al., 2002) (Fig. 1). The 2-amino hydrogen atom of CDAPY interacts with the carboxylate oxygen $\mathrm{O} 1$ of CSA via an $\mathrm{N}-\mathrm{H} \cdots \mathrm{O}$ hydrogen bond forming an $R_{2}^{1}(6)$ ring motif. Thus, the $\mathrm{O} 1$ oxygen atom acts as a trifurcated acceptor. A similar set of three fused rings was observed in the crystal structure of 2,6-diamino-4-chloropyrimidinium 2-carboxy-3-nitrobenzoate (Mohana et al., 2017). However, in compound (I) the role of the 2-amino and 6-amino groups has been reversed. A self-complementary base pairing via a pair of $\mathrm{N} 2-\mathrm{H} \cdots \mathrm{N}^{\mathrm{i}}$ (homosynthon) hydrogen bonds forming an $R_{2}^{2}(8)$ ring motif is also been observed. This type of base pairing is also observed in the crystal structures of 2,6-diamino-4-chloropyridinium 4-carboxybutanoate (Edison et al., 2014), 2,6-diamino-4-chloropyrimidine-benzoic acid (Thanigaimani et al., 2012a) and bis(2,6-diamino-4-chloropyrimidin-1-ium) fumarate (Thanigaimani et al., 2012b). The 2,6-diamino-4-chloropyrimidinium

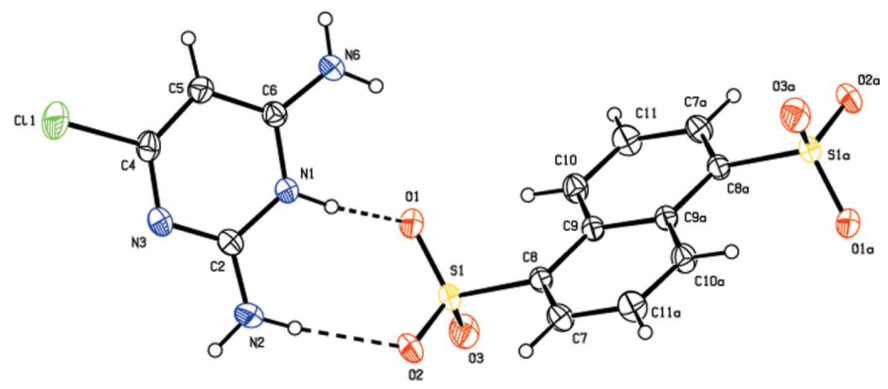

Figure 2

ORTEP view of compound (II), with the atom-numbering scheme. Displacement ellipsoids are drawn at $50 \%$ probability level. Dashed lines represent hydrogen bonds. 
Table 2

Hydrogen-bond geometry $\left(\AA,^{\circ}\right)$ for (II).

\begin{tabular}{lllll}
\hline$D-\mathrm{H} \cdots A$ & $D-\mathrm{H}$ & $\mathrm{H} \cdots A$ & $D \cdots A$ & $D-\mathrm{H} \cdots A$ \\
\hline $\mathrm{N} 1-\mathrm{H} 1 \cdots \mathrm{O} 1$ & 0.86 & 1.92 & $2.708(2)$ & 152 \\
$\mathrm{~N} 2-\mathrm{H} 2 A \cdots \mathrm{O} 2^{\mathrm{i}}$ & 0.86 & 2.08 & $2.868(3)$ & 152 \\
$\mathrm{~N} 2-\mathrm{H} 2 B \cdots \mathrm{O} 2$ & 0.86 & 2.10 & $2.953(2)$ & 174 \\
$\mathrm{~N} 6-\mathrm{H} 6 A \cdots \mathrm{N}^{\mathrm{ii}}$ & 0.86 & 2.25 & $2.943(2)$ & 138 \\
$\mathrm{~N}^{\mathrm{ii}}-\mathrm{H} 6 B \cdots 3^{\mathrm{iii}}$ & 0.86 & 2.01 & $2.808(2)$ & 154 \\
\hline
\end{tabular}

Symmetry codes: (i) $\quad-x+1,-y+1,-z ; \quad$ (ii) $\quad x+\frac{1}{2},-y+\frac{3}{2}, z+\frac{1}{2}$; $\quad$ (iii) $x-\frac{1}{2},-y+\frac{3}{2}, z+\frac{1}{2}$.

5-chlorosalicylate units are linked via a $\mathrm{Cl} \cdots \mathrm{Cl}$ interaction (a type I interaction; Cavallo et al., 2016) with a distance and angle of 3.3505 (12) $\AA$ and 151.37 (10) ${ }^{\circ}$, respectively (Durka et al., 2015) (Fig. 3). Furthermore, a weak $\mathrm{C}-\mathrm{H}$. . O $\mathrm{O}^{\mathrm{iii}}$ hydrogenbonding interaction is present in this crystal structure. In addition, a weak stacking interaction with $C g 1 \cdots C g 2$ [3.6624 (14) A; symmetry code: $x,-1+y, z ; C g 1$ and $C g 2$ are the centroids of the $\mathrm{N} 1 / \mathrm{C} 2 / \mathrm{N} 3 / \mathrm{C} 4 / \mathrm{C} 5 / \mathrm{C} 6$ and $\mathrm{C} 8-\mathrm{C} 13$ rings, respectively] and $\mathrm{C}-\mathrm{Cl} \cdots \pi$ interactions [3.4469 (13) $\AA$ with an angle of $152.24(9)^{\circ}$; symmetry code: $-\frac{1}{2}+x, \frac{1}{2}-y,-\frac{1}{2}+z$ ] (Muthukumaran et al., 2011) further stabilize this crystal structure (Fig. 4).

In salt (II), the sulfonate group mimics the role of the carboxylate oxygen atoms in generating an $R_{2}^{2}(8)$ motif (heterosynthon) involving the aminopyrimidinium cation (CDAPY) (Bernstein et al., 1995; Balasubramani et al., 2007). All units of the CDAPY and NSA ions are hydrogen bonded (Table 2) to generate a quadruple DDAA array with fused ring motifs $R_{2}^{2}(8), R_{4}^{2}(8)$ and $R_{2}^{2}(8)$ (Fig. 5). This type of array has also been reported earlier (Robert et al., 2001; Umadevi et al., 2002; Raj et al., 2003; Subashini et al., 2007; Thanigaimani et al., 2007; Liu \& Chen, 2012). In addition, the NSA anions also generate $R_{3}^{2}(10)$ and $R_{3}^{3}(21)$ ring motifs via $\mathrm{N}-\mathrm{H} \cdots \mathrm{O}$ bonds. Weak $\pi-\pi$ stacking interactions $[C g 1 \cdots C g 4=3.4781$ (11) $\AA$; symmetry code: $\frac{3}{2}-x,-\frac{1}{2}+y, \frac{1}{2}-z$ and $C g 4 \cdots C g 2$

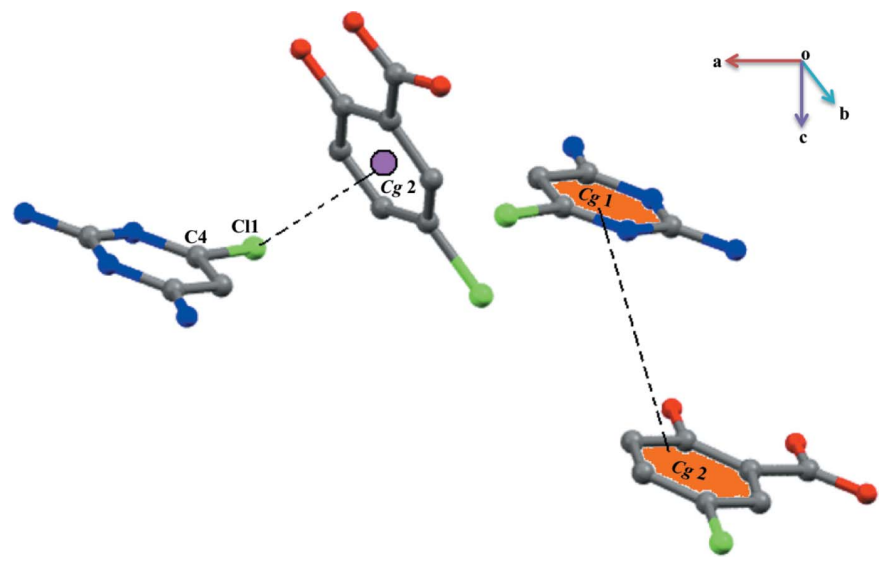

Figure 4

A weak $\mathrm{C}-\mathrm{Cl} \cdots \pi$ interaction and $\pi-\pi$ stacking interactions.

$=3.4781$ (11) $\AA$; symmetry code: $\frac{1}{2}+x, \frac{3}{2}-y, \frac{1}{2}+z ; C g 1, C g 2$ and $\mathrm{Cg} 4$ are the centroids of the $\mathrm{C} 7 / \mathrm{C} 8 / \mathrm{C} 9 / \mathrm{C}^{\prime} / \mathrm{C}^{\prime} 0^{\prime} / \mathrm{C} 11^{\prime}, \mathrm{C} 9 / \mathrm{C} 10 /$ $\mathrm{C} 11 / \mathrm{C}^{\prime} / \mathrm{C}^{\prime} / \mathrm{C} 9^{\prime}$ and $\mathrm{N} 1 / \mathrm{C} 2 / \mathrm{N} 3 / \mathrm{C} 4 / \mathrm{C} 5 / \mathrm{C} 6$ rings, respectively] is also present (Fig. 6).

\section{Database survey}

Various salts of 5-chlorosalicylate have been reported: 2-methylquinolinium 5-chloro-2-hydroxybenzoate (Zhang et al., 2014), 4-amino-5-chloro-2,6-dimethylpyrimidinium 5-chloro-2-hydroxybenzoate (Rajam et al., 2017) and 2-amino4,6-dimethylpyrimidinium 5-chlorosalicylate (Ebenezer \& Muthiah, 2012). Similarly, various salts of half a molecule of naphthalene-1,5-disulfonate have been reported: bis(2-trifluoromethyl-1H-benzimidazole-3-ium) naphthalene-1,5-disulfonate (Liu, 2012), bis(3-methylanilinium) naphthalene1,5-disulfonate (Liu \& Chen, 2012) and bis(2-methylpiperidinium) naphthalene-1,5-disulfonate ( $\mathrm{Xu}, 2012)$.

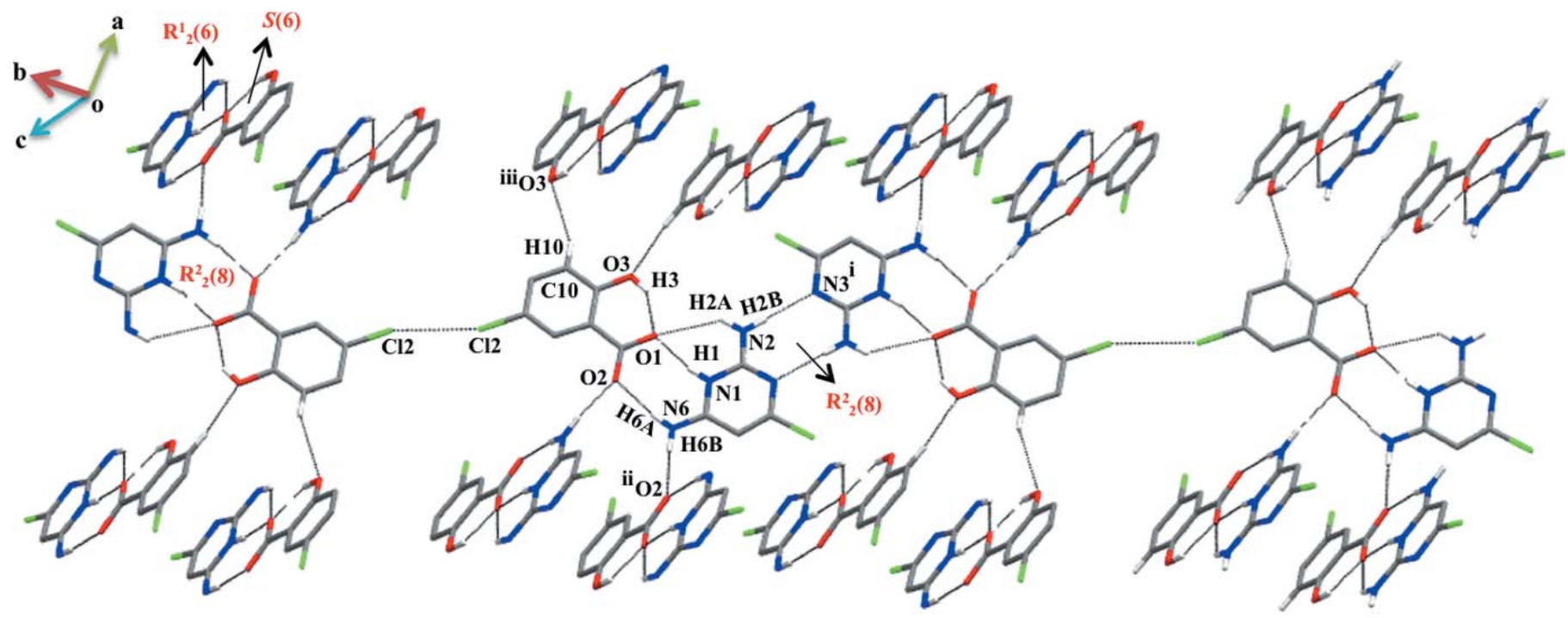

Figure 3

Supramolecular layered structure extended as a chain via $\mathrm{Cl} \cdots \mathrm{Cl}$ interactions in (I). 


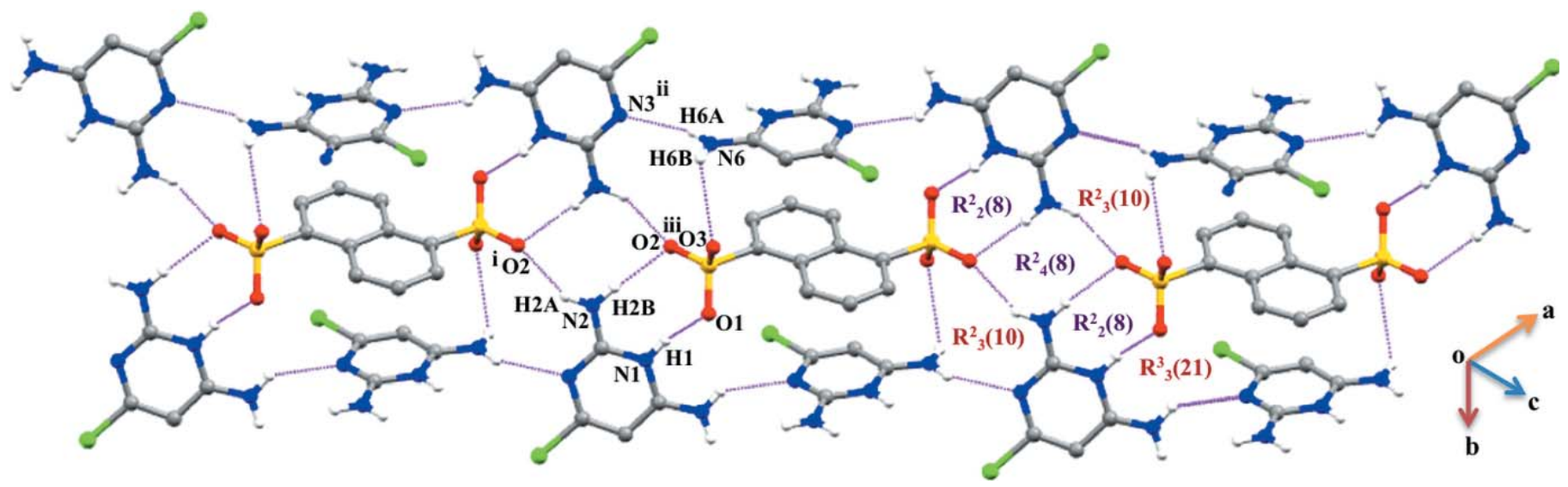

Figure 5

Formation of a quadruple $D D A A$ array in (II) via $\mathrm{N}-\mathrm{H} \cdots \mathrm{O}$ hydrogen bonds.

\section{Synthesis and crystallization}

Compounds (I) and (II) were synthesized by mixing hot ethanolic solutions (1:1) of 2,6-diamino-4-chloropyrimidine (36 mg) with 5-chlorosalicylic acid (43 mg) (I)/naphthalene1,5-disulfonic acid (72 mg) (II). These mixtures were warmed to $333 \mathrm{~K}$ for $25 \mathrm{~min}$. Colourless crystals separated out from the mother liquor at room temperature after a week.

\section{Refinement}

Crystal data, data collection and structure refinement details are summarized in Table 3. All $\mathrm{H}$ atoms were initially located

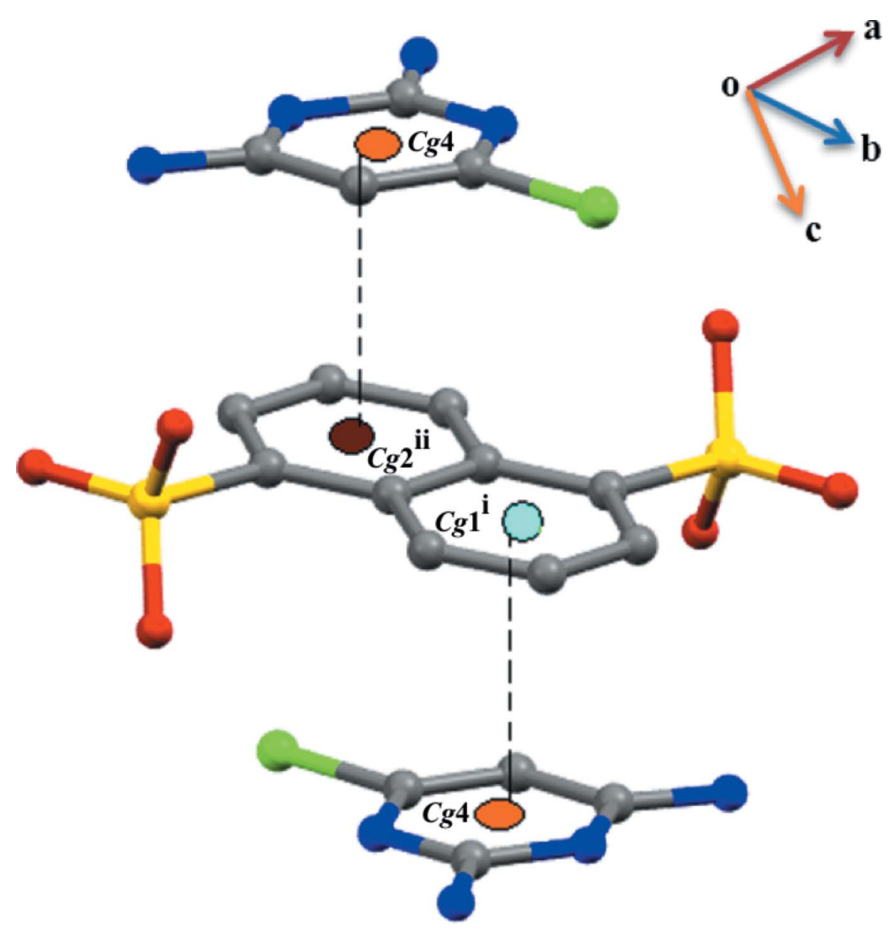

Figure 6

A view of the $\pi-\pi$ stacking interactions between the pyrimidinium cation and the anion. readily in difference-Fourier maps and were treated as riding atoms with $\mathrm{C}-\mathrm{H}=0.93 \AA$ (aromatic), $\mathrm{N}-\mathrm{H}=0.86 \AA$ and $\mathrm{O}-\mathrm{H}=0.82 \AA$ with $U_{\text {iso }}(\mathrm{H})=k U_{\text {eq }}(\mathrm{C}, \mathrm{N}, \mathrm{O})$, where $k=1.5$ for hydroxy and 1.2 for all other $\mathrm{H}$ atoms.

\section{Acknowledgements}

The EN-FIST Centre of Excellence, Ljubljana, Slovenia, is thanked for the use of the SuperNova diffractometer.

\section{Funding information}

RSD thanks the UGC-BSR India for the award of an RFSMS. PTM thanks UGC, New Delhi, for a UGC Emeritus fellowship. FP thanks the Slovenian Research Agency for financial support (PI-0230-0175).

\section{References}

Agilent. (2013). CrysAlis PRO. Agilent Technologies UK Ltd, Yarnton, England.

Baker, B. R. \& Santi, D. V. (1965). J. Pharm. Sci. 54, 1252-1257.

Balasubramani, K., Thomas Muthiah, P. \& Lynch, D. E. (2007). Chem. Cent. J. 1, 28.

Bernstein, J., Davis, R. E., Shimoni, L. \& Chang, N. L. (1995). Angew. Chem. Int. Ed. Engl. 34, 1555-1573.

Cavallo, G., Metrangolo, P., Milani, R., Pilati, T., Priimagi, A., Resnati, G. \& Terraneo, G. (2016). Chem. Rev. 116, 2478-2601.

Durka, K., Kliś, T. \& Serwatowski, J. (2015). Acta Cryst. E71, 14711474.

Ebenezer, S. \& Muthiah, P. T. (2012). Cryst. Growth Des. 12, 37663785.

Edison, B., Balasubramani, K., Thanigaimani, K., Khalib, N. C., Arshad, S. \& Razak, I. A. (2014). Acta Cryst. E70, o857-o858.

Hunt, W. E., Schwalbe, C. H., Bird, K. \& Mallinson, P. D. (1980). Biochem. J. 187, 533-536.

Liu, M.-L. (2012). Acta Cryst. E68, o342.

Liu, M.-L. \& Chen, Z.-Q. (2012). Acta Cryst. E68, o1745.

Macrae, C. F., Bruno, I. J., Chisholm, J. A., Edgington, P. R., McCabe, P., Pidcock, E., Rodriguez-Monge, L., Taylor, R., van de Streek, J. \& Wood, P. A. (2008). J. Appl. Cryst. 41, 466-470.

Mohana, M., Thomas Muthiah, P. \& Butcher, R. J. (2017). Acta Cryst. C73, 536-540.

Muthukumaran, J., Parthiban, A., Kannan, M., Rao, H. S. P. \& Krishna, R. (2011). Acta Cryst. E67, o898-o899. 
Table 3

Experimental details.

(I) (II)

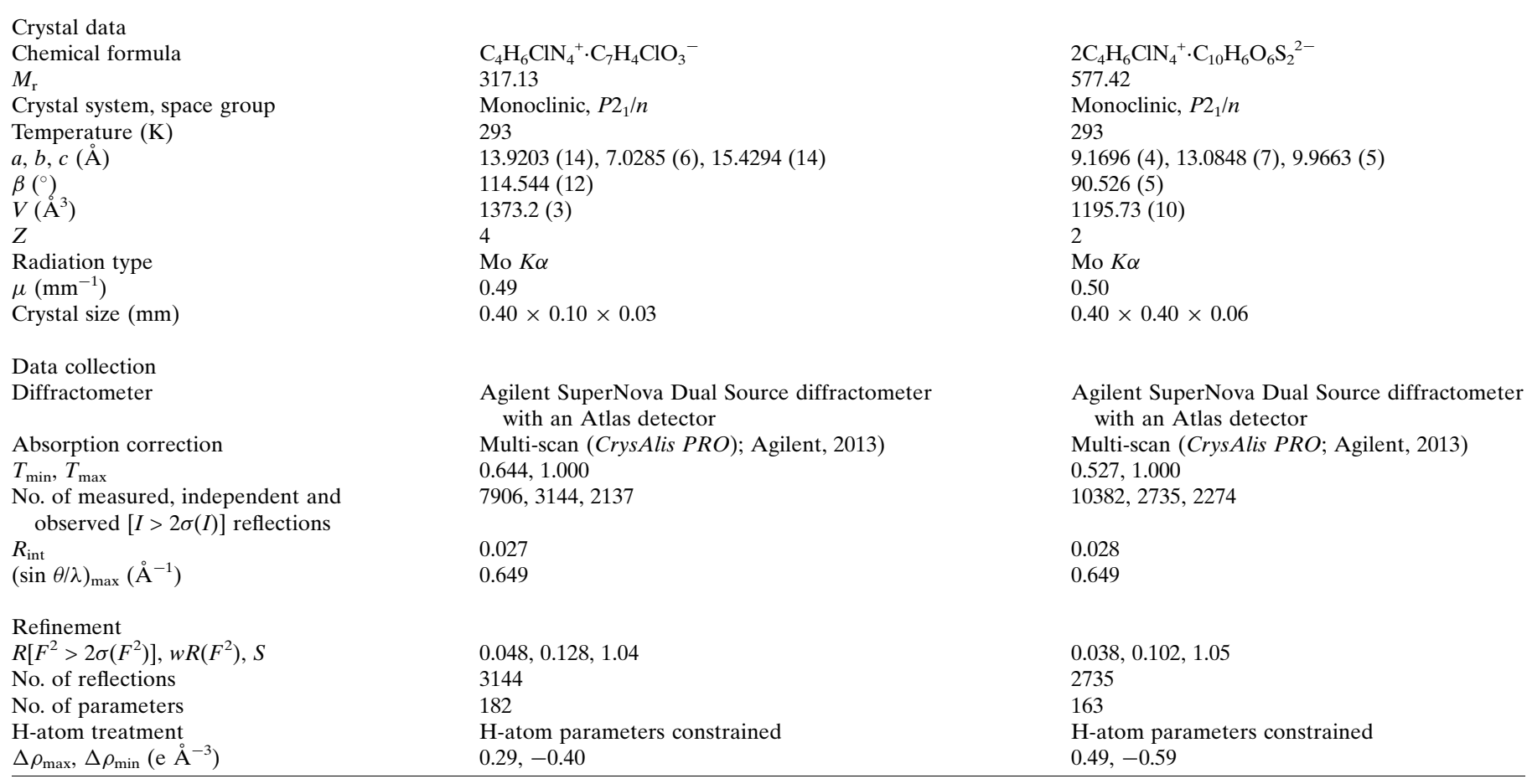

Computer programs: CrysAlis PRO (Agilent, 2013), SHELXT2014 (Sheldrick, 2015a), SHELXL2014 (Sheldrick, 2015b), PLATON (Spek, 2009) and Mercury (Macrae et al., 2008).

Panneerselvam, P., Stanley, N. \& Muthiah, P. T. (2002). Acta Cryst. E58, o180-o182.

Prabakaran, P., Murugesan, S., Muthiah, P. T., Bocelli, G. \& Righi, L. (2001). Acta Cryst. E57, o933-0936.

Raj, S. B., Muthiah, P. T., Rychlewska, U. \& Warzajtis, B. (2003). CrystEngComm, 5, 48-53.

Rajam, A., Muthiah, P. T., Butcher, R. J., Jasinski, J. P. \& Glidewell, C. (2017). Acta Cryst. C73, 862-868.

Robert, J. J., Raj, S. B. \& Muthiah, P. T. (2001). Acta Cryst. E57, o1206-o1208.

Sheldrick, G. M. (2015a). Acta Cryst. A71, 3-8.

Sheldrick, G. M. (2015b). Acta Cryst. C71, 3-8.

Spek, A. L. (2009). Acta Cryst. D65, 148-155.
Subashini, A., Muthiah, P. T., Bocelli, G. \& Cantoni, A. (2007). Acta Cryst. E63, o3775.

Thanigaimani, K., Khalib, N. C., Arshad, S. \& Razak, I. A. (2012a). Acta Cryst. E68, o3442-03443.

Thanigaimani, K., Khalib, N. C., Farhadikoutenaei, A., Arshad, S. \& Razak, I. A. (2012b). Acta Cryst. E68, o3321-o3322.

Thanigaimani, K., Muthiah, P. T. \& Lynch, D. E. (2007). Acta Cryst. E63, o4555-o4556.

Umadevi, B., Prabakaran, P. \& Muthiah, P. T. (2002). Acta Cryst. C58, o510-0512.

Xu, Q. (2012). Acta Cryst. E68, o1733.

Zhang, J., Jin, S., Tao, L., Liu, B. \& Wang, D. (2014). J. Mol. Struct. 1072, 208-220. 


\section{supporting information}

Acta Cryst. (2018). E74, 237-241 [https://doi.org/10.1107/S2056989018001196]

Supramolecular interactions in 2,6-diamino-4-chloropyrimidin-1-ium 5-chlorosalicylate and bis(2,6-diamino-4-chloropyrimidin-1-ium) naphthalene-1,5-disulfonate

\section{Robert Swinton Darious, Packianathan Thomas Muthiah and Franc Perdih}

Computing details

For both structures, data collection: CrysAlis PRO (Agilent, 2013); cell refinement: CrysAlis PRO (Agilent, 2013); data reduction: CrysAlis PRO (Agilent, 2013); program(s) used to solve structure: SHELXT2014 (Sheldrick, 2015a); program(s) used to refine structure: SHELXL2014 (Sheldrick, 2015b); molecular graphics: PLATON (Spek, 2009), Mercury (Macrae et al., 2008); software used to prepare material for publication: PLATON (Spek, 2009).

2,6-Diamino-4-chloropyrimidin-1-ium 2-chloro-6-hydroxybenzoate (I)

Crystal data

$\mathrm{C}_{4} \mathrm{H}_{6} \mathrm{ClN}_{4}{ }^{+} \cdot \mathrm{C}_{7} \mathrm{H}_{4} \mathrm{ClO}_{3}^{-}$

$M_{r}=317.13$

Monoclinic, $P 2_{1} / n$

$a=13.9203(14) \AA$

$b=7.0285(6) \AA$

$c=15.4294(14) \AA$

$\beta=114.544(12)^{\circ}$

$V=1373.2(3) \AA^{3}$

$Z=4$

Data collection

Agilent SuperNova Dual Source diffractometer with an Atlas detector

Radiation source: SuperNova (Mo) X-ray Source

Mirror monochromator

Detector resolution: 10.4933 pixels $\mathrm{mm}^{-1}$

$\omega$ scans

Absorption correction: multi-scan

(CrysAlis PRO); Agilent, 2013)

\section{Refinement}

Refinement on $F^{2}$

Least-squares matrix: full

$R\left[F^{2}>2 \sigma\left(F^{2}\right)\right]=0.048$

$w R\left(F^{2}\right)=0.128$

$S=1.04$

3144 reflections
$F(000)=648$

$D_{\mathrm{x}}=1.534 \mathrm{Mg} \mathrm{m}^{-3}$

Mo $K \alpha$ radiation, $\lambda=0.71073 \AA$

Cell parameters from 1734 reflections

$\theta=3.9-27.5^{\circ}$

$\mu=0.49 \mathrm{~mm}^{-1}$

$T=293 \mathrm{~K}$

Needle, colorless

$0.40 \times 0.10 \times 0.03 \mathrm{~mm}$

$T_{\min }=0.644, T_{\max }=1.000$

7906 measured reflections

3144 independent reflections

2137 reflections with $I>2 \sigma(I)$

$R_{\text {int }}=0.027$

$\theta_{\max }=27.5^{\circ}, \theta_{\min }=2.9^{\circ}$

$h=-18 \rightarrow 17$

$k=-7 \rightarrow 9$

$l=-20 \rightarrow 19$

182 parameters

0 restraints

Hydrogen site location: inferred from neighbouring sites

$\mathrm{H}$-atom parameters constrained 
$w=1 /\left[\sigma^{2}\left(F_{\mathrm{o}}^{2}\right)+(0.0482 P)^{2}+0.5033 P\right]$

where $P=\left(F_{\mathrm{o}}^{2}+2 F_{\mathrm{c}}^{2}\right) / 3$

$(\Delta / \sigma)_{\max }<0.001$

$$
\Delta \rho_{\max }=0.29 \mathrm{e} \AA^{-3}
$$

\section{Special details}

Geometry. All esds (except the esd in the dihedral angle between two 1.s. planes) are estimated using the full covariance matrix. The cell esds are taken into account individually in the estimation of esds in distances, angles and torsion angles; correlations between esds in cell parameters are only used when they are defined by crystal symmetry. An approximate (isotropic) treatment of cell esds is used for estimating esds involving l.s. planes.

Fractional atomic coordinates and isotropic or equivalent isotropic displacement parameters $\left(\hat{A}^{2}\right)$

\begin{tabular}{|c|c|c|c|c|}
\hline & $x$ & $y$ & $z$ & $U_{\text {iso }} * / U_{\text {eq }}$ \\
\hline $\mathrm{Cl1}$ & $0.25461(6)$ & $-0.27636(11)$ & $-0.00641(5)$ & $0.0674(2)$ \\
\hline $\mathrm{N} 1$ & $0.38557(14)$ & $0.2707(3)$ & $0.12207(13)$ & $0.0386(4)$ \\
\hline $\mathrm{H} 1$ & 0.4128 & 0.3792 & 0.1450 & $0.046^{*}$ \\
\hline N2 & $0.50448(17)$ & $0.2485(3)$ & $0.05504(16)$ & $0.0566(6)$ \\
\hline $\mathrm{H} 2 \mathrm{~B}$ & 0.5313 & 0.1895 & 0.0215 & $0.068^{*}$ \\
\hline $\mathrm{H} 2 \mathrm{~A}$ & 0.5293 & 0.3573 & 0.0796 & $0.068^{*}$ \\
\hline N3 & $0.38700(15)$ & $0.0032(3)$ & $0.03064(13)$ & $0.0442(5)$ \\
\hline N6 & $0.27205(16)$ & $0.3072(3)$ & $0.19347(15)$ & $0.0503(5)$ \\
\hline H6A & 0.3023 & 0.4141 & 0.2157 & $0.060 *$ \\
\hline H6B & 0.2207 & 0.2687 & 0.2063 & $0.060^{*}$ \\
\hline $\mathrm{C} 2$ & $0.42485(18)$ & $0.1715(3)$ & $0.06889(16)$ & $0.0404(5)$ \\
\hline $\mathrm{C} 4$ & $0.30522(17)$ & $-0.0606(3)$ & $0.04713(16)$ & $0.0414(5)$ \\
\hline $\mathrm{C} 5$ & $0.26096(16)$ & $0.0273(3)$ & $0.09993(15)$ & $0.0398(5)$ \\
\hline H5 & 0.2048 & -0.0266 & 0.1089 & $0.048^{*}$ \\
\hline C6 & $0.30401(16)$ & $0.2033(3)$ & $0.14025(15)$ & $0.0368(5)$ \\
\hline $\mathrm{Cl} 2$ & $0.50995(6)$ & $1.29096(12)$ & $0.45221(5)$ & $0.0745(3)$ \\
\hline $\mathrm{O} 1$ & $0.49261(13)$ & $0.5917(2)$ & $0.18881(13)$ & $0.0534(5)$ \\
\hline $\mathrm{O} 2$ & $0.38734(12)$ & $0.6467(2)$ & $0.26174(12)$ & $0.0501(4)$ \\
\hline $\mathrm{O} 3$ & $0.62982(14)$ & $0.8382(3)$ & $0.19368(15)$ & $0.0617(5)$ \\
\hline H3 & 0.5981 & 0.7365 & 0.1805 & $0.093 *$ \\
\hline $\mathrm{C} 7$ & $0.46259(17)$ & $0.6930(3)$ & $0.24184(16)$ & $0.0393(5)$ \\
\hline $\mathrm{C} 8$ & $0.51962(15)$ & $0.8751(3)$ & $0.27862(15)$ & $0.0362(5)$ \\
\hline C9 & $0.60003(17)$ & $0.9377(4)$ & $0.25339(17)$ & $0.0437(6)$ \\
\hline $\mathrm{C} 10$ & $0.65092(19)$ & $1.1105(4)$ & 0.28915 (19) & $0.0563(7)$ \\
\hline H10 & 0.7038 & 1.1527 & 0.2718 & $0.068^{*}$ \\
\hline $\mathrm{C} 11$ & $0.6235(2)$ & $1.2181(4)$ & $0.34943(19)$ & $0.0580(7)$ \\
\hline H11 & 0.6576 & 1.3330 & 0.3729 & $0.070^{*}$ \\
\hline $\mathrm{C} 12$ & $0.54500(19)$ & $1.1548(4)$ & $0.37507(17)$ & $0.0488(6)$ \\
\hline $\mathrm{C} 13$ & $0.49410(17)$ & $0.9865(3)$ & $0.34066(16)$ & $0.0412(5)$ \\
\hline H13 & 0.4417 & 0.9458 & 0.3590 & $0.049 *$ \\
\hline
\end{tabular}

Atomic displacement parameters $\left(\AA^{2}\right)$

\begin{tabular}{lllllll}
\hline & $U^{11}$ & $U^{22}$ & $U^{33}$ & $U^{12}$ & $U^{13}$ & $U^{23}$ \\
\hline C11 & $0.0648(4)$ & $0.0561(5)$ & $0.0783(5)$ & $-0.0240(3)$ & $0.0267(4)$ & $-0.0292(4)$ \\
N1 & $0.0432(10)$ & $0.0303(10)$ & $0.0503(10)$ & $-0.0045(8)$ & $0.0274(9)$ & $-0.0059(9)$
\end{tabular}




$\begin{array}{lllllll}\mathrm{N} 2 & 0.0670(13) & 0.0480(13) & 0.0795(15) & -0.0198(11) & 0.0551(12) & -0.0247(12) \\ \mathrm{N} 3 & 0.0479(11) & 0.0401(11) & 0.0490(11) & -0.0089(9) & 0.0245(9) & -0.0100(10) \\ \mathrm{N} 6 & 0.0527(11) & 0.0418(12) & 0.0747(14) & -0.0065(10) & 0.0448(11) & -0.0087(11) \\ \text { C2 } & 0.0460(12) & 0.0383(13) & 0.0442(12) & -0.0032(11) & 0.0258(10) & -0.0027(11) \\ \text { C4 } & 0.0403(12) & 0.0346(13) & 0.0421(11) & -0.0045(10) & 0.0099(10) & -0.0031(11) \\ \text { C5 } & 0.0337(11) & 0.0385(13) & 0.0477(12) & -0.0050(10) & 0.0176(10) & 0.0007(11) \\ \text { C6 } & 0.0354(11) & 0.0341(12) & 0.0433(11) & 0.0029(9) & 0.0187(10) & 0.0030(10) \\ \text { C12 } & 0.0751(5) & 0.0685(5) & 0.0735(5) & 0.0006(4) & 0.0245(4) & -0.0334(4) \\ \text { O1 } & 0.0566(10) & 0.0395(10) & 0.0798(12) & -0.0057(8) & 0.0439(9) & -0.0171(9) \\ \text { O2 } & 0.0519(10) & 0.0380(9) & 0.0762(11) & -0.0081(8) & 0.0425(9) & -0.0081(9) \\ \text { O3 } & 0.0555(11) & 0.0592(13) & 0.0892(13) & -0.0074(9) & 0.0487(10) & -0.0126(11) \\ \text { C7 } & 0.0401(12) & 0.0314(12) & 0.0494(12) & 0.0037(10) & 0.0216(10) & 0.0012(10) \\ \text { C8 } & 0.0315(10) & 0.0326(12) & 0.0427(11) & 0.0022(9) & 0.0136(9) & 0.0001(10) \\ \text { C9 } & 0.0351(11) & 0.0427(14) & 0.0539(13) & 0.0011(10) & 0.0192(10) & 0.0002(12) \\ \text { C10 } & 0.0436(13) & 0.0566(17) & 0.0695(16) & -0.0126(13) & 0.0242(13) & -0.0008(15) \\ \text { C11 } & 0.0517(15) & 0.0456(15) & 0.0638(16) & -0.0121(13) & 0.0112(13) & -0.0105(14) \\ \text { C12 } & 0.0451(13) & 0.0436(14) & 0.0485(13) & 0.0009(11) & 0.0102(11) & -0.0088(12) \\ \text { C13 } & 0.0356(11) & 0.0399(13) & 0.0466(12) & 0.0005(10) & 0.0156(10) & -0.0032(11)\end{array}$

Geometric parameters ( $\left.\AA,{ }^{\circ}\right)$

\begin{tabular}{llll}
\hline $\mathrm{C} 11-\mathrm{C} 4$ & $1.731(2)$ & $\mathrm{C} 2-\mathrm{C} 12$ & $1.747(3)$ \\
$\mathrm{N} 1-\mathrm{C} 2$ & $1.353(3)$ & $\mathrm{O} 1-\mathrm{C} 7$ & $1.279(3)$ \\
$\mathrm{N} 1-\mathrm{C} 6$ & $1.362(3)$ & $\mathrm{O} 2-\mathrm{C} 7$ & $1.251(3)$ \\
$\mathrm{N} 1-\mathrm{H} 1$ & 0.8600 & $\mathrm{O} 3-\mathrm{C} 9$ & $1.352(3)$ \\
$\mathrm{N} 2-\mathrm{C} 2$ & $1.328(3)$ & $\mathrm{O} 3-\mathrm{H} 3$ & 0.8200 \\
$\mathrm{~N} 2-\mathrm{H} 2 \mathrm{~B}$ & 0.8600 & $\mathrm{C} 7-\mathrm{C} 8$ & $1.488(3)$ \\
$\mathrm{N} 2-\mathrm{H} 2 \mathrm{~A}$ & 0.8600 & $\mathrm{C} 8-\mathrm{C} 13$ & $1.392(3)$ \\
$\mathrm{N} 3-\mathrm{C} 2$ & $1.329(3)$ & $\mathrm{C} 8-\mathrm{C} 9$ & $1.400(3)$ \\
$\mathrm{N} 3-\mathrm{C} 4$ & $1.342(3)$ & $\mathrm{C} 9-\mathrm{C} 10$ & $1.399(4)$ \\
$\mathrm{N} 6-\mathrm{C} 6$ & $1.307(3)$ & $\mathrm{C} 10-\mathrm{C} 11$ & $1.370(4)$ \\
$\mathrm{N} 6-\mathrm{H} 6 \mathrm{~A}$ & 0.8600 & $\mathrm{C} 10-\mathrm{H} 10$ & 0.9300 \\
$\mathrm{~N} 6-\mathrm{H} 6 \mathrm{~B}$ & 0.8600 & $\mathrm{C} 11-\mathrm{C} 12$ & $1.382(4)$ \\
$\mathrm{C} 4-\mathrm{C} 5$ & $1.357(3)$ & $\mathrm{C} 11-\mathrm{H} 11$ & 0.9300 \\
$\mathrm{C} 5-\mathrm{C} 6$ & $1.402(3)$ & $\mathrm{C} 12-\mathrm{C} 13$ & $1.368(3)$ \\
$\mathrm{C} 5-\mathrm{H} 5$ & 0.9300 & $\mathrm{C} 13-\mathrm{H} 13$ & 0.9300 \\
& & & 109.5 \\
$\mathrm{C} 2-\mathrm{N} 1-\mathrm{C} 6$ & $121.80(19)$ & $\mathrm{C} 9-\mathrm{O} 3-\mathrm{H} 3$ & $122.8(2)$ \\
$\mathrm{C} 2-\mathrm{N} 1-\mathrm{H} 1$ & 119.1 & $\mathrm{O} 2-\mathrm{C} 7-\mathrm{O} 1$ & $119.9(2)$ \\
$\mathrm{C} 6-\mathrm{N} 1-\mathrm{H} 1$ & 119.1 & $\mathrm{O} 2-\mathrm{C} 7-\mathrm{C} 8$ & $117.29(19)$ \\
$\mathrm{C} 2-\mathrm{N} 2-\mathrm{H} 2 \mathrm{~B}$ & 120.0 & $\mathrm{O} 1-\mathrm{C} 7-\mathrm{C} 8$ & $118.4(2)$ \\
$\mathrm{C} 2-\mathrm{N} 2-\mathrm{H} 2 \mathrm{~A}$ & 120.0 & $\mathrm{C} 13-\mathrm{C} 8-\mathrm{C} 9$ & $119.85(19)$ \\
$\mathrm{H} 2 \mathrm{~B}-\mathrm{N} 2-\mathrm{H} 2 \mathrm{~A}$ & 120.0 & $\mathrm{C} 13-\mathrm{C} 8-\mathrm{C} 7$ & $121.7(2)$ \\
$\mathrm{C} 2-\mathrm{N} 3-\mathrm{C} 4$ & $115.1(2)$ & $\mathrm{C} 9-\mathrm{C} 8-\mathrm{C} 7$ & $118.0(2)$ \\
$\mathrm{C} 6-\mathrm{N} 6-\mathrm{H} 6 \mathrm{~A}$ & 120.0 & $\mathrm{O} 3-\mathrm{C} 9-\mathrm{C} 10$ & $122.3(2)$ \\
$\mathrm{C} 6-\mathrm{N} 6-\mathrm{H} 6 \mathrm{~B}$ & 120.0 & $\mathrm{O} 3-\mathrm{C} 9-\mathrm{C} 8$ & $119.7(2)$ \\
$\mathrm{H} 6 \mathrm{~A}-\mathrm{N} 6-\mathrm{H} 6 \mathrm{~B}$ & 120.0 & $\mathrm{C} 11-\mathrm{C} 10-\mathrm{C} 9$ & $120.6(2)$ \\
$\mathrm{N} 2-\mathrm{C} 2-\mathrm{N} 3$ & $119.6(2)$ & & \\
& & &
\end{tabular}




$\begin{array}{llll}\mathrm{N} 2-\mathrm{C} 2-\mathrm{N} 1 & 117.5(2) & \mathrm{C} 11-\mathrm{C} 10-\mathrm{H} 10 & 119.7 \\ \mathrm{~N} 3-\mathrm{C} 2-\mathrm{N} 1 & 122.8(2) & \mathrm{C} 9-\mathrm{C} 10-\mathrm{H} 10 & 119.7 \\ \mathrm{~N} 3-\mathrm{C} 4-\mathrm{C} 5 & 126.4(2) & \mathrm{C} 10-\mathrm{C} 11-\mathrm{C} 12 & 119.5(2) \\ \mathrm{N} 3-\mathrm{C} 4-\mathrm{C} 11 & 114.28(18) & \mathrm{C} 10-\mathrm{C} 11-\mathrm{H} 11 & 120.2 \\ \mathrm{C} 5-\mathrm{C} 4-\mathrm{C} 11 & 119.28(18) & \mathrm{C} 12-\mathrm{C} 11-\mathrm{H} 11 & 120.2 \\ \mathrm{C} 4-\mathrm{C} 5-\mathrm{C} 6 & 116.8(2) & \mathrm{C} 13-\mathrm{C} 12-\mathrm{C} 11 & 120.7(2) \\ \mathrm{C} 4-\mathrm{C} 5-\mathrm{H} 5 & 121.6 & \mathrm{C} 13-\mathrm{C} 12-\mathrm{C} 12 & 119.5(2) \\ \mathrm{C} 6-\mathrm{C} 5-\mathrm{H} 5 & 121.6 & \mathrm{C} 11-\mathrm{C} 12-\mathrm{C} 12 & 119.8(2) \\ \mathrm{N} 6-\mathrm{C} 6-\mathrm{N} 1 & 117.7(2) & \mathrm{C} 12-\mathrm{C} 13-\mathrm{C} 8 & 121.0(2) \\ \mathrm{N} 6-\mathrm{C} 6-\mathrm{C} 5 & 125.3(2) & \mathrm{C} 12-\mathrm{C} 13-\mathrm{H} 13 & 119.5 \\ \mathrm{~N} 1-\mathrm{C} 6-\mathrm{C} 5 & 117.0(2) & \mathrm{C} 8-\mathrm{C} 13-\mathrm{H} 13 & 119.5 \\ & & & \\ \mathrm{C} 4-\mathrm{N} 3-\mathrm{C} 2-\mathrm{N} 2 & -178.7(2) & \mathrm{O} 1-\mathrm{C} 7-\mathrm{C} 8-\mathrm{C} 9 & 179.9(2) \\ \mathrm{C} 4-\mathrm{N} 3-\mathrm{C} 2-\mathrm{N} 1 & 1.2(3) & \mathrm{C} 13-\mathrm{C} 8-\mathrm{C} 9-\mathrm{O} 3 & 0.7(3) \\ \mathrm{C} 6-\mathrm{N} 1-\mathrm{C} 2-\mathrm{N} 2 & -180.0(2) & \mathrm{C} 7-\mathrm{C} 8-\mathrm{C} 9-\mathrm{O} 3 & -1.2(3) \\ \mathrm{C} 6-\mathrm{N} 1-\mathrm{C} 2-\mathrm{N} 3 & 0.2(3) & \mathrm{C} 13-\mathrm{C} 8-\mathrm{C} 9-\mathrm{C} 10 & 179.5(2) \\ \mathrm{C} 2-\mathrm{N} 3-\mathrm{C} 4-\mathrm{C} 5 & -1.7(3) & \mathrm{C} 7-\mathrm{C} 8-\mathrm{C} 9-\mathrm{C} 10 & 179.5(2) \\ \mathrm{C} 2-\mathrm{N} 3-\mathrm{C} 4-\mathrm{C} 11 & 178.08(16) & \mathrm{O} 3-\mathrm{C} 9-\mathrm{C} 10-\mathrm{C} 11 & 0.7(4) \\ \mathrm{N} 3-\mathrm{C} 4-\mathrm{C} 5-\mathrm{C} 6 & 0.9(3) & \mathrm{C} 8-\mathrm{C} 9-\mathrm{C} 10-\mathrm{C} 11 & 0.1(4) \\ \mathrm{C} 11-\mathrm{C} 4-\mathrm{C} 5-\mathrm{C} 6 & -178.93(16) & \mathrm{C} 9-\mathrm{C} 10-\mathrm{C} 11-\mathrm{C} 12 & -0.2(4) \\ \mathrm{C} 2-\mathrm{N} 1-\mathrm{C} 6-\mathrm{N} 6 & 179.0(2) & \mathrm{C} 10-\mathrm{C} 11-\mathrm{C} 12-\mathrm{C} 13 & 179.8(2) \\ \mathrm{C} 2-\mathrm{N} 1-\mathrm{C} 6-\mathrm{C} 5 & -1.1(3) & \mathrm{C} 10-\mathrm{C} 11-\mathrm{C} 12-\mathrm{C} 12 & -0.3(4) \\ \mathrm{C} 4-\mathrm{C} 5-\mathrm{C} 6-\mathrm{N} 6 & -179.5(2) & \mathrm{C} 11-\mathrm{C} 12-\mathrm{C} 13-\mathrm{C} 8 & 179.61(17) \\ \mathrm{C} 4-\mathrm{C} 5-\mathrm{C} 6-\mathrm{N} 1 & 0.6(3) & \mathrm{C} 12-\mathrm{C} 12-\mathrm{C} 13-\mathrm{C} 8 & 1.1(3) \\ \mathrm{O} 2-\mathrm{C} 7-\mathrm{C} 8-\mathrm{C} 13 & 4.7(3) & \mathrm{C} 9-\mathrm{C} 8-\mathrm{C} 13-\mathrm{C} 12 & -179.7(2) \\ \mathrm{O} 1-\mathrm{C} 7-\mathrm{C} 8-\mathrm{C} 13 & -176.68(19) & \mathrm{C} 7-\mathrm{C} 8-\mathrm{C} 13-\mathrm{C} 12 & \\ \mathrm{O} 2-\mathrm{C} 7-\mathrm{C} 8-\mathrm{C} 9 & -176.1(2) & & \end{array}$

Hydrogen-bond geometry $\left(\AA,{ }^{\circ}\right)$

\begin{tabular}{lllll}
\hline$D-\mathrm{H} \cdots A$ & $D-\mathrm{H}$ & $\mathrm{H} \cdots A$ & $D \cdots A$ & $D-\mathrm{H} \cdots A$ \\
\hline $\mathrm{N} 1-\mathrm{H} 1 \cdots \mathrm{O} 1$ & 0.86 & 1.82 & $2.664(3)$ & 168 \\
$\mathrm{~N} 2-\mathrm{H} 2 A \cdots \mathrm{O} 1$ & 0.86 & 2.56 & $3.223(3)$ & 135 \\
$\mathrm{~N} 2-\mathrm{H} 2 B \cdots \mathrm{N}^{\mathrm{i}}$ & 0.86 & 2.13 & $2.970(3)$ & 165 \\
$\mathrm{O} 3-\mathrm{H} 3 \cdots \mathrm{O} 1$ & 0.82 & 1.83 & $2.557(3)$ & 146 \\
$\mathrm{~N} 6-\mathrm{H} 6 A \cdots \mathrm{O} 2$ & 0.86 & 1.97 & $2.824(3)$ & 172 \\
$\mathrm{~N} 6-\mathrm{H} 6 B \cdots \mathrm{O} 2$ & 0.86 & 1.96 & $2.819(3)$ & 172 \\
$\mathrm{C} 10-\mathrm{H} 10 \cdots{ }^{i i}$ & 0.93 & 2.51 & $3.358(4)$ & 151
\end{tabular}

Symmetry codes: (i) $-x+1,-y,-z$; (ii) $-x+1 / 2, y-1 / 2,-z+1 / 2$; (iii) $-x+3 / 2, y+1 / 2,-z+1 / 2$.

Bis(2,6-diamino-4-chloropyrimidin-1-ium) naphthalene-1,5-disulfonate (II)

Crystal data

$2 \mathrm{C}_{4} \mathrm{H}_{6} \mathrm{ClN}_{4}^{+} \cdot \mathrm{C}_{10} \mathrm{H}_{6} \mathrm{O}_{6} \mathrm{~S}_{2}{ }^{2-}$

$M_{r}=577.42$

Monoclinic, $P 2_{1} / n$

$a=9.1696(4) \AA$

$b=13.0848(7) \AA$

$c=9.9663(5) \AA$ $\beta=90.526(5)^{\circ}$

$V=1195.73(10) \AA^{3}$

$Z=2$

$F(000)=592$

$D_{\mathrm{x}}=1.604 \mathrm{Mg} \mathrm{m}^{-3}$

Mo $K \alpha$ radiation, $\lambda=0.71073 \AA$ 
Cell parameters from 3749 reflections

$\theta=3.7-30.1^{\circ}$

$\mu=0.50 \mathrm{~mm}^{-1}$

\section{Data collection}

Agilent SuperNova Dual Source diffractometer with an Atlas detector

Radiation source: SuperNova (Mo) X-ray Source

Mirror monochromator

Detector resolution: 10.4933 pixels $\mathrm{mm}^{-1}$

$\omega$ scans

Absorption correction: multi-scan

(CrysAlis PRO; Agilent, 2013)

\section{Refinement}

Refinement on $F^{2}$

Least-squares matrix: full

$R\left[F^{2}>2 \sigma\left(F^{2}\right)\right]=0.038$

$w R\left(F^{2}\right)=0.102$

$S=1.05$

2735 reflections

163 parameters

0 restraints
$T=293 \mathrm{~K}$

Prism, colorless

$0.40 \times 0.40 \times 0.06 \mathrm{~mm}$

$T_{\min }=0.527, T_{\max }=1.000$

10382 measured reflections

2735 independent reflections

2274 reflections with $I>2 \sigma(I)$

$R_{\text {int }}=0.028$

$\theta_{\text {max }}=27.5^{\circ}, \theta_{\min }=3.0^{\circ}$

$h=-8 \rightarrow 11$

$k=-16 \rightarrow 15$

$l=-12 \rightarrow 12$

Hydrogen site location: inferred from neighbouring sites

$\mathrm{H}$-atom parameters constrained

$w=1 /\left[\sigma^{2}\left(F_{\mathrm{o}}^{2}\right)+(0.0444 P)^{2}+0.5881 P\right]$

where $P=\left(F_{\mathrm{o}}^{2}+2 F_{\mathrm{c}}^{2}\right) / 3$

$(\Delta / \sigma)_{\max }<0.001$

$\Delta \rho_{\max }=0.49 \mathrm{e} \AA^{-3}$

$\Delta \rho_{\min }=-0.59$ e $\AA^{-3}$

Special details

Geometry. All esds (except the esd in the dihedral angle between two 1.s. planes) are estimated using the full covariance matrix. The cell esds are taken into account individually in the estimation of esds in distances, angles and torsion angles; correlations between esds in cell parameters are only used when they are defined by crystal symmetry. An approximate (isotropic) treatment of cell esds is used for estimating esds involving l.s. planes.

Fractional atomic coordinates and isotropic or equivalent isotropic displacement parameters $\left(\AA^{2}\right)$

\begin{tabular}{lllll}
\hline & $x$ & $y$ & $z$ & $U_{\text {iso }} * / U_{\text {eq }}$ \\
\hline C11 & $0.10474(6)$ & $0.91005(5)$ & $0.13497(8)$ & $0.0693(2)$ \\
N1 & $0.47951(17)$ & $0.72194(12)$ & $0.24106(15)$ & $0.0379(4)$ \\
H1 & 0.5541 & 0.6839 & 0.2572 & $0.045^{*}$ \\
N2 & $0.4259(2)$ & $0.61789(16)$ & $0.0635(2)$ & $0.0675(7)$ \\
H2A & 0.3734 & 0.6006 & -0.0047 & $0.081^{*}$ \\
H2B & 0.5013 & 0.5823 & 0.0853 & $0.081^{*}$ \\
N3 & $0.27559(18)$ & $0.75496(14)$ & $0.10314(17)$ & $0.0437(4)$ \\
N6 & $0.54208(19)$ & $0.81915(14)$ & $0.42350(17)$ & $0.0444(4)$ \\
H6A & 0.6148 & 0.7789 & 0.4376 & $0.053^{*}$ \\
H6B & 0.5275 & 0.8701 & 0.4761 & $0.053^{*}$ \\
C2 & $0.3908(2)$ & $0.69909(16)$ & $0.1347(2)$ & $0.0420(5)$ \\
C4 & $0.2528(2)$ & $0.83590(15)$ & $0.1817(2)$ & $0.0393(4)$ \\
C5 & $0.3328(2)$ & $0.86453(15)$ & $0.2911(2)$ & $0.0377(4)$ \\
H5 & 0.3089 & 0.9215 & 0.3422 & $0.045^{*}$ \\
C6 & $0.45350(19)$ & $0.80277(14)$ & $0.32183(18)$ & $0.0333(4)$ \\
S1 & $0.80386(5)$ & $0.55638(4)$ & $0.21480(4)$ & $0.03723(15)$ \\
O1 & $0.75993(16)$ & $0.65802(11)$ & $0.25770(15)$ & $0.0491(4)$ \\
O2 & $0.68017(17)$ & $0.49952(12)$ & $0.15999(15)$ & $0.0517(4)$
\end{tabular}




$\begin{array}{lllll}\text { O3 } & 0.92854(18) & 0.55687(13) & 0.12748(14) & 0.0541(4) \\ \text { C7 } & 0.7936(2) & 0.40041(15) & 0.39206(19) & 0.0379(4) \\ \text { H7 } & 0.7184 & 0.3761 & 0.3375 & 0.045^{*} \\ \text { C8 } & 0.86088(18) & 0.49007(14) & 0.36151(17) & 0.0307(4) \\ \text { C9 } & 0.97829(18) & 0.52894(13) & 0.44251(17) & 0.0293(4) \\ \text { C10 } & 1.0523(2) & 0.62150(15) & 0.41375(19) & 0.0385(4) \\ \text { H10 } & 1.0250 & 0.6595 & 0.3388 & 0.046^{*} \\ \text { C11 } & 1.1626(2) & 0.65540(16) & 0.4944(2) & 0.0427(5) \\ \text { H11 } & 1.2097 & 0.7163 & 0.4739 & 0.051^{*}\end{array}$

Atomic displacement parameters $\left(\AA^{2}\right)$

\begin{tabular}{lllllll}
\hline & $U^{11}$ & $U^{22}$ & $U^{33}$ & $U^{12}$ & $U^{13}$ & $U^{23}$ \\
\hline C11 & $0.0446(3)$ & $0.0740(4)$ & $0.0888(5)$ & $0.0223(3)$ & $-0.0221(3)$ & $-0.0052(4)$ \\
N1 & $0.0362(8)$ & $0.0366(8)$ & $0.0405(8)$ & $0.0056(7)$ & $-0.0128(7)$ & $-0.0061(7)$ \\
N2 & $0.0709(13)$ & $0.0633(13)$ & $0.0676(13)$ & $0.0230(11)$ & $-0.0364(11)$ & $-0.0346(11)$ \\
N3 & $0.0371(9)$ & $0.0485(10)$ & $0.0454(9)$ & $0.0019(7)$ & $-0.0144(7)$ & $-0.0041(8)$ \\
N6 & $0.0459(10)$ & $0.0451(9)$ & $0.0420(9)$ & $0.0073(8)$ & $-0.0150(8)$ & $-0.0107(7)$ \\
C2 & $0.0415(11)$ & $0.0420(11)$ & $0.0424(10)$ & $0.0010(8)$ & $-0.0122(9)$ & $-0.0066(8)$ \\
C4 & $0.0275(9)$ & $0.0429(11)$ & $0.0474(11)$ & $0.0012(8)$ & $-0.0047(8)$ & $0.0052(9)$ \\
C5 & $0.0347(9)$ & $0.0367(10)$ & $0.0416(10)$ & $0.0032(8)$ & $-0.0022(8)$ & $-0.0028(8)$ \\
C6 & $0.0327(9)$ & $0.0338(9)$ & $0.0333(9)$ & $-0.0023(7)$ & $-0.0023(7)$ & $0.0000(7)$ \\
S1 & $0.0411(3)$ & $0.0401(3)$ & $0.0303(2)$ & $0.0063(2)$ & $-0.00909(19)$ & $0.00095(18)$ \\
O1 & $0.0499(8)$ & $0.0425(8)$ & $0.0545(9)$ & $0.0128(7)$ & $-0.0185(7)$ & $-0.0033(7)$ \\
O2 & $0.0563(9)$ & $0.0532(9)$ & $0.0451(8)$ & $0.0030(7)$ & $-0.0252(7)$ & $-0.0052(7)$ \\
O3 & $0.0625(10)$ & $0.0643(10)$ & $0.0356(8)$ & $0.0090(8)$ & $0.0075(7)$ & $0.0121(7)$ \\
C7 & $0.0326(9)$ & $0.0426(11)$ & $0.0382(10)$ & $-0.0054(8)$ & $-0.0048(8)$ & $-0.0017(8)$ \\
C8 & $0.0295(8)$ & $0.0349(9)$ & $0.0276(8)$ & $0.0026(7)$ & $-0.0017(7)$ & $0.0004(7)$ \\
C9 & $0.0279(8)$ & $0.0325(9)$ & $0.0276(8)$ & $0.0019(7)$ & $-0.0002(6)$ & $0.0012(7)$ \\
C10 & $0.0415(10)$ & $0.0380(10)$ & $0.0361(9)$ & $-0.0029(8)$ & $-0.0033(8)$ & $0.0086(8)$ \\
C11 & $0.0437(11)$ & $0.0387(10)$ & $0.0456(11)$ & $-0.0133(8)$ & $-0.0023(9)$ & $0.0073(8)$ \\
& & & & & &
\end{tabular}

Geometric parameters $\left(A,{ }^{\circ}\right)$

\begin{tabular}{llll}
\hline $\mathrm{C} 11-\mathrm{C} 4$ & $1.7290(19)$ & $\mathrm{S} 1-\mathrm{O} 3$ & $1.4431(16)$ \\
$\mathrm{N} 1-\mathrm{C} 6$ & $1.352(2)$ & $\mathrm{S} 1-\mathrm{O} 1$ & $1.4550(15)$ \\
$\mathrm{N} 1-\mathrm{C} 2$ & $1.363(2)$ & $\mathrm{S} 1-\mathrm{O} 2$ & $1.4584(15)$ \\
$\mathrm{N} 1-\mathrm{H} 1$ & 0.8600 & $\mathrm{~S} 1-\mathrm{C} 8$ & $1.7749(17)$ \\
$\mathrm{N} 2-\mathrm{C} 2$ & $\mathrm{C} 7-\mathrm{C} 8$ & $1.361(3)$ \\
$\mathrm{N} 2-\mathrm{H} 2 \mathrm{~A}$ & $1.319(3)$ & $\mathrm{C} 7-\mathrm{C} 11^{\mathrm{i}}$ & $1.403(3)$ \\
$\mathrm{N} 2-\mathrm{H} 2 \mathrm{~B}$ & 0.8600 & $\mathrm{C} 7-\mathrm{H} 7$ & 0.9300 \\
$\mathrm{~N} 3-\mathrm{C} 2$ & 0.8600 & $\mathrm{C} 8-\mathrm{C} 9$ & $1.433(2)$ \\
$\mathrm{N} 3-\mathrm{C} 4$ & $1.321(3)$ & $\mathrm{C} 9-\mathrm{C} 10$ & $1.419(3)$ \\
$\mathrm{N} 6-\mathrm{C} 6$ & $1.335(3)$ & $\mathrm{C} 9-\mathrm{C} 9^{\mathrm{i}}$ & $1.427(3)$ \\
$\mathrm{N} 6-\mathrm{H} 6 \mathrm{~A}$ & $1.311(2)$ & $\mathrm{C} 10-\mathrm{C} 11$ & $1.361(3)$ \\
$\mathrm{N} 6-\mathrm{H} 6 \mathrm{~B}$ & 0.8600 & $\mathrm{C} 10-\mathrm{H} 10$ & 0.9300 \\
$\mathrm{C} 4-\mathrm{C} 5$ & 0.8600 & $\mathrm{C} 11-\mathrm{C} 7^{\mathrm{i}}$ & $1.403(3)$ \\
$\mathrm{C} 5-\mathrm{C} 6$ & $1.361(3)$ & $\mathrm{C} 11-\mathrm{H} 11$ & 0.9300
\end{tabular}




\begin{tabular}{|c|c|c|c|}
\hline $\mathrm{C} 5-\mathrm{H} 5$ & 0.9300 & & \\
\hline $\mathrm{C} 6-\mathrm{N} 1-\mathrm{C} 2$ & $121.84(16)$ & $\mathrm{O} 3-\mathrm{S} 1-\mathrm{O} 1$ & $113.34(10)$ \\
\hline $\mathrm{C} 6-\mathrm{N} 1-\mathrm{H} 1$ & 119.1 & $\mathrm{O} 3-\mathrm{S} 1-\mathrm{O} 2$ & $113.21(10)$ \\
\hline $\mathrm{C} 2-\mathrm{N} 1-\mathrm{H} 1$ & 119.1 & $\mathrm{O} 1-\mathrm{S} 1-\mathrm{O} 2$ & $111.10(9)$ \\
\hline $\mathrm{C} 2-\mathrm{N} 2-\mathrm{H} 2 \mathrm{~A}$ & 120.0 & $\mathrm{O} 3-\mathrm{S} 1-\mathrm{C} 8$ & $105.66(8)$ \\
\hline $\mathrm{C} 2-\mathrm{N} 2-\mathrm{H} 2 \mathrm{~B}$ & 120.0 & $\mathrm{O} 1-\mathrm{S} 1-\mathrm{C} 8$ & $106.56(8)$ \\
\hline $\mathrm{H} 2 \mathrm{~A}-\mathrm{N} 2-\mathrm{H} 2 \mathrm{~B}$ & 120.0 & $\mathrm{O} 2-\mathrm{S} 1-\mathrm{C} 8$ & $106.34(9)$ \\
\hline $\mathrm{C} 2-\mathrm{N} 3-\mathrm{C} 4$ & $115.40(16)$ & $\mathrm{C} 8-\mathrm{C} 7-\mathrm{C} 11^{\mathrm{i}}$ & $120.15(17)$ \\
\hline $\mathrm{C} 6-\mathrm{N} 6-\mathrm{H} 6 \mathrm{~A}$ & 120.0 & $\mathrm{C} 8-\mathrm{C} 7-\mathrm{H} 7$ & 119.9 \\
\hline $\mathrm{C} 6-\mathrm{N} 6-\mathrm{H} 6 \mathrm{~B}$ & 120.0 & $\mathrm{C} 11^{\mathrm{i}}-\mathrm{C} 7-\mathrm{H} 7$ & 119.9 \\
\hline $\mathrm{H} 6 \mathrm{~A}-\mathrm{N} 6-\mathrm{H} 6 \mathrm{~B}$ & 120.0 & $\mathrm{C} 7-\mathrm{C} 8-\mathrm{C} 9$ & $121.31(16)$ \\
\hline $\mathrm{N} 2-\mathrm{C} 2-\mathrm{N} 3$ & $121.08(18)$ & $\mathrm{C} 7-\mathrm{C} 8-\mathrm{S} 1$ & $118.35(13)$ \\
\hline $\mathrm{N} 2-\mathrm{C} 2-\mathrm{N} 1$ & $116.65(18)$ & $\mathrm{C} 9-\mathrm{C} 8-\mathrm{S} 1$ & $120.31(13)$ \\
\hline $\mathrm{N} 3-\mathrm{C} 2-\mathrm{N} 1$ & $122.27(18)$ & $\mathrm{C} 10-\mathrm{C} 9-\mathrm{C} 9^{\mathrm{i}}$ & $119.00(19)$ \\
\hline $\mathrm{N} 3-\mathrm{C} 4-\mathrm{C} 5$ & $127.02(18)$ & $\mathrm{C} 10-\mathrm{C} 9-\mathrm{C} 8$ & $123.19(15)$ \\
\hline $\mathrm{N} 3-\mathrm{C} 4-\mathrm{Cl} 1$ & $114.47(14)$ & $\mathrm{C} 9-\mathrm{C} 9-\mathrm{C} 8$ & $117.8(2)$ \\
\hline $\mathrm{C} 5-\mathrm{C} 4-\mathrm{Cl1}$ & $118.51(16)$ & $\mathrm{C} 11-\mathrm{C} 10-\mathrm{C} 9$ & $120.90(17)$ \\
\hline $\mathrm{C} 4-\mathrm{C} 5-\mathrm{C} 6$ & $115.80(18)$ & $\mathrm{C} 11-\mathrm{C} 10-\mathrm{H} 10$ & 119.6 \\
\hline $\mathrm{C} 4-\mathrm{C} 5-\mathrm{H} 5$ & 122.1 & $\mathrm{C} 9-\mathrm{C} 10-\mathrm{H} 10$ & 119.6 \\
\hline $\mathrm{C} 6-\mathrm{C} 5-\mathrm{H} 5$ & 122.1 & $\mathrm{C} 10-\mathrm{C} 11-\mathrm{C}^{\mathrm{i}}$ & $120.83(18)$ \\
\hline $\mathrm{N} 6-\mathrm{C} 6-\mathrm{N} 1$ & $118.48(17)$ & $\mathrm{C} 10-\mathrm{C} 11-\mathrm{H} 11$ & 119.6 \\
\hline $\mathrm{N} 6-\mathrm{C} 6-\mathrm{C} 5$ & $123.87(18)$ & $\mathrm{C} 7^{\mathrm{i}}-\mathrm{C} 11-\mathrm{H} 11$ & 119.6 \\
\hline $\mathrm{N} 1-\mathrm{C} 6-\mathrm{C} 5$ & $117.64(16)$ & & \\
\hline $\mathrm{C} 4-\mathrm{N} 3-\mathrm{C} 2-\mathrm{N} 2$ & $-179.1(2)$ & $\mathrm{O} 3-\mathrm{S} 1-\mathrm{C} 8-\mathrm{C} 7$ & $-116.17(16)$ \\
\hline $\mathrm{C} 4-\mathrm{N} 3-\mathrm{C} 2-\mathrm{N} 1$ & $0.7(3)$ & $\mathrm{O} 1-\mathrm{S} 1-\mathrm{C} 8-\mathrm{C} 7$ & $122.99(16)$ \\
\hline $\mathrm{C} 6-\mathrm{N} 1-\mathrm{C} 2-\mathrm{N} 2$ & $-179.4(2)$ & $\mathrm{O} 2-\mathrm{S} 1-\mathrm{C} 8-\mathrm{C} 7$ & $4.41(18)$ \\
\hline $\mathrm{C} 6-\mathrm{N} 1-\mathrm{C} 2-\mathrm{N} 3$ & $0.8(3)$ & $\mathrm{O} 3-\mathrm{S} 1-\mathrm{C} 8-\mathrm{C} 9$ & $61.75(17)$ \\
\hline $\mathrm{C} 2-\mathrm{N} 3-\mathrm{C} 4-\mathrm{C} 5$ & $-1.8(3)$ & $\mathrm{O} 1-\mathrm{S} 1-\mathrm{C} 8-\mathrm{C} 9$ & $-59.08(16)$ \\
\hline $\mathrm{C} 2-\mathrm{N} 3-\mathrm{C} 4-\mathrm{Cl} 1$ & $178.00(16)$ & $\mathrm{O} 2-\mathrm{S} 1-\mathrm{C} 8-\mathrm{C} 9$ & $-177.67(14)$ \\
\hline $\mathrm{N} 3-\mathrm{C} 4-\mathrm{C} 5-\mathrm{C} 6$ & $1.3(3)$ & $\mathrm{C} 7-\mathrm{C} 8-\mathrm{C} 9-\mathrm{C} 10$ & $179.44(18)$ \\
\hline $\mathrm{C} 11-\mathrm{C} 4-\mathrm{C} 5-\mathrm{C} 6$ & $-178.48(14)$ & $\mathrm{S} 1-\mathrm{C} 8-\mathrm{C} 9-\mathrm{C} 10$ & $1.6(2)$ \\
\hline $\mathrm{C} 2-\mathrm{N} 1-\mathrm{C} 6-\mathrm{N} 6$ & $178.92(19)$ & $\mathrm{C} 7-\mathrm{C} 8-\mathrm{C} 9-\mathrm{C} 9^{\mathrm{i}}$ & $-0.6(3)$ \\
\hline $\mathrm{C} 2-\mathrm{N} 1-\mathrm{C} 6-\mathrm{C} 5$ & $-1.3(3)$ & $\mathrm{S} 1-\mathrm{C} 8-\mathrm{C} 9-\mathrm{C} 9^{\mathrm{i}}$ & $-178.50(17)$ \\
\hline $\mathrm{C} 4-\mathrm{C} 5-\mathrm{C} 6-\mathrm{N} 6$ & $-179.95(19)$ & $\mathrm{C} 9-\mathrm{C} 9-\mathrm{C} 10-\mathrm{C} 11$ & $-0.3(3)$ \\
\hline $\mathrm{C} 4-\mathrm{C} 5-\mathrm{C} 6-\mathrm{N} 1$ & $0.3(3)$ & $\mathrm{C} 8-\mathrm{C} 9-\mathrm{C} 10-\mathrm{C} 11$ & $179.62(19)$ \\
\hline $\mathrm{C} 11^{\mathrm{i}}-\mathrm{C} 7-\mathrm{C} 8-\mathrm{C} 9$ & $0.8(3)$ & $\mathrm{C} 9-\mathrm{C} 10-\mathrm{C} 11-\mathrm{C} 7^{\mathrm{i}}$ & $0.1(3)$ \\
\hline $\mathrm{C} 11^{\mathrm{i}}-\mathrm{C} 7-\mathrm{C} 8-\mathrm{S} 1$ & $178.73(16)$ & & \\
\hline
\end{tabular}

Symmetry code: (i) $-x+2,-y+1,-z+1$.

Hydrogen-bond geometry $\left(A,{ }^{\circ}\right)$

\begin{tabular}{lllll}
\hline$D-\mathrm{H} \cdots A$ & $D-\mathrm{H}$ & $\mathrm{H} \cdots A$ & $D \cdots A$ & $D-\mathrm{H} \cdots A$ \\
\hline $\mathrm{N} 1-\mathrm{H} 1 \cdots \mathrm{O} 1$ & 0.86 & 1.92 & $2.708(2)$ & 152 \\
$\mathrm{~N} 2-\mathrm{H} 2 A \cdots \mathrm{O} 2{ }^{\mathrm{ii}}$ & 0.86 & 2.08 & $2.868(3)$ & 152 \\
$\mathrm{~N} 2-\mathrm{H} 2 B \cdots \mathrm{O} 2$ & 0.86 & 2.10 & $2.953(2)$ & 174
\end{tabular}


supporting information

$\mathrm{N} 6-\mathrm{H} 6 A \cdots \mathrm{N} 3^{\mathrm{iii}} \quad 0.86$

$\begin{array}{ll}0.86 & 2.25 \\ 0.86 & \end{array}$

2.01

$2.943(2)$

$2.808(2)$

138

$\mathrm{N} 6-\mathrm{H} 6 B \cdots \mathrm{O} 3^{\text {iv }}$

0.86

Symmetry codes: (ii) $-x+1,-y+1,-z$; (iii) $x+1 / 2,-y+3 / 2, z+1 / 2$; (iv) $x-1 / 2,-y+3 / 2, z+1 / 2$. 